\title{
CARACTERIZACIÓN DEL SUBSECTOR DE LA ALFARERÍA Y CERÁMICA EN LOS MUNICIPIOS DE RÁQUIRA Y LA CHAMBA PARA LA COMERCIALIZACIÓN INTERNACIONAL DE ARTESANÍAS COLOMBIANAS
}

\author{
CHARACTERIZATION OF THE POTTERY AND \\ CERAMIC SUBSECTOR FOR THE INTERNATIONAL \\ COMMERCIALIZATION OF COLOMBIAN \\ HANDICRAFTS IN THE MUNICIPALITIES OF RAQUIRA \\ AND THE CHAMBA
}

Álvaro Lamprea Delgado ${ }^{1}$

\begin{abstract}
RESUMEN
La ausencia de conocimiento por parte de los artesanos y de sus organizaciones sobre el desarrollo de estrategias de comercialización a nivel nacional e internacional ha impedido el crecimiento y la mejora en la competitividad del subsector. Por lo anterior, se requiere de la creación y la expansión de empresas con mentalidad y conciencia exportadora y la necesidad de mejorar la cadena productiva para garantizar un constante suministro de productos que faciliten cumplir con los pedidos en el exterior. Se debe transformar el sistema tradicional de producción en uno más productivo permitiendoles competir y acceder a los mercados internacionales, así como crear mecanismos que promuevan procesos de producción y comercialización, a una escala sostenible y viable. El estudio efectuado en los municipios de mayor producción cerámica del país, Ráquira, en el departa-
\end{abstract}

\footnotetext{
${ }^{1}$ Profesional en Comercio Internacional, Especialista en Gerencia de Mercadeo, Docente Investigador, Ingeniería Comercial, Universidad de Ciencias Aplicadas y Ambientales U.D.C.A. Dirección para correspondencia e-mail: alamprea@udca.edu.co
}

mento de Boyacá y La Chamba, en el departamento del Tolima, desarrolló la caracterización y la descripción del sector. Esta es una investigación aplicada de carácter cuantitativo y cualitativo de tipo histórico, en la cual se examinaron aspectos como el análisis del sector y del producto artesanal, la determinación y la priorización de factores relevantes, análisis dofa y la fijación de objetivos estratégicos de acuerdo a las perspectivas. Se buscó describir y discutir los avances y los desarrollos del subsector para aumentar la oferta exportable de artesanías de productos cerámicos y/o alfareros, que logren un alto impacto en la generación de empleo y la consecución de divisas y que mejoren el nivel de vida de los artesanos.

Palabras clave: Artesano, caracterización, comercialización internacional, cerámica, alfarería.

\section{SUMMARY}

The lack of knowlegde by handicraftsmen and their organizations about the development of commercial- 
ization strategies at national and international level has hindered the growth and improvement of the competitivity of this artisan subsector. Therefore, the creation and development of companies with exportation mentality and consciousness and the necessity to improve the productive chain to guarantee a constant product flux to fulfill the external demands is necessary. The traditional system has to be transformed in a more productive and competitive one to grant to compete and to accede to international markets, as well as to develop strategies that promote production processes at a sustainable and viable scale. The study, carried out in the municipalities of the main ceramic production in the country, "Ráquira", in the deparment of Boyaca and "The Chamba", in the deparment of Tolima, developed the characterization and description of the subsector. In this applied research of quantitative and qualitative historical type, aspects such as the sector's and artisan's products analysis, the determination and priorization of relevant factors, a Dofa analysis and the fixation of strategist objectives were examined. The evolution and development of the subsector was described and discussed in order to increase the exportable supply of ceramic products and attain a high impact in the generation of employment and consecution of holdings and therefore in the quality of life of the handicraftsmen.

Key words: handicraftsmen, characterization, international commercialization, pottery, ceramics.

\section{INTRODUCCIÓN}

Los municipios de Ráquira y La Chamba fueron los principales centros olleros indígenas del país y en la actualidad preservan parte de las técnicas originarias de sus antepasados, logrando que sus artículos tengan un importante valor cultural y comercial. La producción artesanal, se desarrolla en zonas rurales y urbanas, teniendo su origen en parcelas campesinas que generan ingresos insuficientes y absorben la mano de obra familiar creando una producción manufacturera incipiente (Artesanías de Colombia, 1999). La producción urbana, se promueve en empresas familiares y, excepcionalmente, en empresas asociativas, tipificadas por una baja capacidad administrativa, principalmente por ser el artesano, quien desarrolla todas las actividades en su taller (Delgado, 2001). Adicionalmente, se caracteriza por una baja acumulación de capital, falta de innovación tecnológica e imperfecciones en los procesos productivos y en los productos (Rodríguez, 2001), factores que dificultan la inserción de estas pequeñas unidades en una economía competitiva, cada vez más globalizada y exigente.

Con el presente estudio, se pretendió caracterizar y analizar este subsector para proporcionar las bases para la construcción de un modelo de comercialización internacional y aumentar la oferta exportable de artesanías de productos cerámicos y/o alfareros, con un alto impacto en la generación de empleo y la consecución de divisas, para mejorar el nivel de vida de los artesanos del subsector cerámico en Colombia. Para esto, se escogió el sector de los municipios de Ráquira (Boyacá) y La Chamba (Tolima).

El municipio de Ráquira ha venido trabajando en diferentes programas de formación empresarial, con el fin de alcanzar un mejor desarrollo del subsector (Ministerio de Desarrollo Económico, 1998a). Algunas de los logros obtenidos fueron el interés de los artesanos por aumentar la producción con excelente calidad para los mercados externos, a través de alianzas estratégicas, la conciencia sobre la necesidad de unir esfuerzos para alcanzar la competitividad y la disposición para constituir la asociación de exportadores de Ráquira; un mayor interés en torno al tema de la contaminación y la participación en la rueda de negocios con compradores.

La diferencia entre el comportamiento de la artesanía rural y la urbana es notoria, en las veredas esta actividad es considera como una ocupación secundaria, figurando la agricultura como una actividad principal (Benavides, 1997), mientras que en el casco urbano los artesanos se dedican exclusivamente al trabajo de la cerámica. Ellos cuentan con técnicas y formas de producción cada vez más sofisticadas, ya que tienen acceso a medios que les facilita el desarrollo de su actividad. Generalmente son los hombres quienes dirigen los talleres, los cuales cuentan con mano de obra ocupada y no perteneciente a la familia (Delgado, 2001).

En cuanto a la producción, una de las dificultades es el aspecto laboral, debido a que la mano de obra ocupada carece de participación constante en el proceso. De los seis días normales de trabajo, solamente laboran cuatro, lo cual perjudica enormemente la producción (Urrutia, 1971). En los últimos años, se está presentando el caso 
de traslado de la producción de las veredas al centro del Municipio, creándose así mayores fuentes de empleo.

En la segunda zona, objeto de estudio, La Chamba (Tolima), donde se han desarrollado importantes proyectos para crear y organizar la cadena productiva de la cerámica, obteniendo logros más exitosos que en el municipio de Ráquira. Entre las ventajas más importantes, se destacan la asociatividad y la consecución de la materia prima, el barro que se extrae de una mina propiedad de la comunidad y lo cual redunda en unos menores costos. La mayoría de talleres son administrados por mujeres, siendo éste quizás uno de los factores que incida en el mayor nivel de asociatividad en La Chamba. Es un importante centro de producción que trabaja la cerámica desde 1930, siendo una de sus características más notorias y favorables los colores y los diseños de sus productos. Existe la producción con diseños tradicionales, pero además se están impulsando nuevos diseños por solicitud de sus clientes, los intermediarios (Navarro, 2001). Las actividades artesanales, se combinan con otras labores, como el jornaleo agrícola, el comercio y la sastrería, que complementan el sustento de la unidad familiar. Dada la importancia de la agricultura durante las épocas de siembra y de la cosecha, se denota una baja en la mano de obra (Rojas, 1984).

El estudio realizado fue aplicado, cuantitativo y cualitativo de tipo histórico. Se determinó la evolución y el auge de la oferta exportable de los productos cerámicos. Además, se buscó conocer si el impacto generado en el crecimiento de la producción mejora la calidad de vida de los artesanos.

\section{MATERIALES Y MÉTODOS}

En Ráquira, se realizaron 30 encuestas y 30 entrevistas personales en profundidad a los artesanos, en las cuales se analizaron principalmente la producción y las ventas directas o indirectas efectuadas hacia los mercados internacionales. Las encuestas, se aplicaron en las veredas de El Centro (nueve talleres), Pueblo Viejo (diez talleres), Candelaria (dos talleres), Santa Bárbara (tres talleres) y los Pinos (tres talleres). Todos los artesanos encuestados en este municipio, se dedican exclusivamente a la actividad artesanal cerámica.

En La Chamba, se efectuaron sondeos y 25 entrevistas en profundidad. Se tuvieron en cuenta principalmente aspectos como el taller, la actividad, el número de empleados, el ingreso y la actividad exportadora. Respecto a este último punto, se buscó conocer el porcentaje de la producción que se dedica hacia la exportación, los países destino, la periodicidad en compras para exportaciones, los vínculos gremiales o asociativos, los tipos de productos, los canales de distribución, la competencia, la logística, el proceso de producción y el diseño. Un alto porcentaje de los lugares de trabajo analizados son de propiedad de las artesanas, así que en cada vivienda existe una unidad de producción, lo que significa un lento aumento de nuevas unidades productivas.

\section{RESULTADOS Y DISCUSIÓN}

Descripción y análisis del subsector cerámico / alfarero en el municipio de Ráquira: El análisis de las encuestas demostró que los ingresos para los 30 talleres no incrementaronsignificativamente al aumentar la producción y tampoco mejoró sustancialmente la calidad de vida de los artesanos. El desarrollo de las exportaciones de productos cerámicos y/o alfareros no ha beneficiado directamente al artesano - productor. Como se observa en el Cuadro 1, el máximo ingreso corresponde a ocho salarios mínimos mensuales, obtenidos por tres talleres, mientras que tres talleres subsisten con medio salario mínimo. Se destaca que, tanto los talleres con mayores ingresos, así como los de menores ingresos, son manejados por sus propietarios, lo cual indica una posible falta de disposición de técnicas y de tácticas de producción y de comercialización de los artesanos de bajos ingresos.

Los intermediarios minoristas y mayoristas que acopian las mercancías en la zona, para luego distribuirlas a los diferentes mercados locales y ocasionalmente para su exportación, se reducen a seis comerciantes. De ellos, uno comercializa gran parte de la producción, por que cuenta con mano de obra disponible y transporte propio. La calidad y los diseños de los productos cerámicos son dos aspectos esenciales e importantes que determinan el valor del producto por su diseño original. En México, por ejemplo (Estage, 1998), la característica "hecho a mano" proporciona una valoración artística y monetaria a los productos, lo mismo ocurre en Ráquira.

Los aspectos de diversificación, las economías de escala y la variedad de líneas de productos están siendo muy 
Cuadro 1. Ingresos de los artesanos medidos en Salario Mínimo Legal (S.M.L.) año 2004 (Ráquira).

\begin{tabular}{|c|c|c|c|}
\hline \multirow{2}{*}{ S.M.L.* } & \multirow{2}{*}{$\begin{array}{c}\text { Número de } \\
\text { Talleres }\end{array}$} & \multicolumn{2}{|c|}{ Propietario del taller } \\
\cline { 3 - 4 } & & SI & NO \\
\hline 0,5 S.M.L. & 3 & 3 & - \\
\hline 1 S.M.L. & 6 & 4 & 2 \\
\hline 1,5 S.M.L. & 3 & 3 & - \\
\hline 2 S.M.L. & 9 & 8 & 1 \\
\hline 2,5 S.M.L. & 6 & 6 & - \\
\hline 8 S.M.L. & 3 & 3 & - \\
\hline TOT ALES & 30 & 27 & 3 \\
\hline
\end{tabular}

Encuesta U.D.C.A, 2004

descuidados, lo que afecta el precio. Se venden volúmenes, pero no calidad y creatividad, como parte del valor agregado. Los productores no cuentan con un método adecuado para calcular los costos de producción, ni su margen de utilidad. La producción en las veredas, en muchas ocasiones, es una labor irregular que no genera los ingresos necesarios (Cuadro 1) para cubrir las necesidades básicas, como ya lo había detectado Fonseca (1985) y lo cual no ha sufrido cambio sustancial en los últimos 20 años. Además, el precio de venta, establecido de acuerdo con la oferta y la demanda del mercado, no permite una acumulación de capital y de excedentes para reinvertir y lograr beneficios.

Los precios son impuestos por los intermediarios mayoristas, sin tener en cuenta los costos de producción de cada taller y con frecuencia el comprador, es decir, un mayorista, determina el precio de los productos por debajo del costo de producción. El artesano, ante esta situación, se tiene que someter (Ministerio de Desarrollo Económico, 1998a), de lo contrario, se le dificultará vender sus productos. El 71\% (21 artesanos) vende su producto a los compradores mayoristas, el 19\% (seis artesanos), a los minoristas que acopian la mercancía para ser vendida en los almacenes ubicados en la ciudad y $10 \%$ (tres artesanos) utiliza la modalidad de venta directa a pequeños compradores, generalmente, turistas. Esta operación, se realiza en el mismo lugar de trabajo, favoreciendo al artesano quien no descuida así la producción.
Los principales países destino de las exportaciones de productos cerámicos y alfareros son los Estados Unidos, Aruba, Israel, Panamá y Ecuador. A través de la Asociación de Artesanos ASOAGUABUENA, se pudo corroborar que sí existe demanda para este tipo de productos para fines de exportación. Esta asociación participó en MANOFACTO 2002, feria de artesanos organizada por Artesanías de Colombia, en la "Plaza de los Artesanos", en el mes de agosto de 2002, en la ciudad de Bogotá, consiguiendo potenciales compradores en el exterior, entre los cuales se destacaron Estados Unidos, Canadá, Israel y Costa Rica, pero no se cumplió con la demanda, debido al manejo inadecuado de la negociación, pues los artesanos no se responsabilizaron por los compromisos adquiridos. Adicionalmente, cada taller costeaba con diferentes precios de venta y costos distintos, lo cual no permitió unificar, de manera organizada, los pedidos.

Como se observa en el Cuadro 2 los artesanos destinan parte o la totalidad de su producción a la exportación. Ningún taller la realiza en forma directa, pues todos dependen y lo hacen a través de los comercializadores mayoristas quienes les compran a precios bajos parte de la producción con destino a los mercados internacionales. Lo anterior genera un alto grado de insatisfacción y frustración entre los artesanos, que aspiran a lograr mayores ventas y, por ende, mejorar sus ingresos.

Las materas en sus diferentes tamaños, diseños y formas, como ya lo detectó Villareal (2000), constituyen 
Cuadro 2. Potencial de la actividad exportadora de artesanías (Ráquira)

\begin{tabular}{|c|c|c|c|}
\hline $\begin{array}{c}\text { PORCENTAJE DE LA } \\
\text { PRODUCCION DEDICADA } \\
\text { A EXPORTAR }^{*}\end{array}$ & $\begin{array}{c}\text { Número de } \\
\text { Talleres }\end{array}$ & \multicolumn{2}{|c|}{ ACTIMDAD EXPORTADORA } \\
\cline { 3 - 4 } & & SI (INTERMEDIARIOS) & NO \\
\hline $0 \%$ & 6 & & 6 \\
\hline $10 \%$ & 3 & 3 & \\
\hline $50 \%$ & 6 & 6 & \\
\hline $80 \%$ & 12 & 12 & \\
\hline $100 \%$ & 3 & 3 & \\
\hline TOTAL & 30 & 24 & 6 \\
\hline
\end{tabular}

Encuesta U.D.C.A, 2004

*Compras hechas por comercializadores para exportar. Año 2004

uno de los productos con mayor demanda, por lo cual, gran porcentaje de talleres se dedica exclusivamente a su producción, sin embargo insuficiente para cubrir las necesidades del mercado, en términos de calidad, de diseño y de entregas a tiempo. Se detecta la especialización alcanzada por algunos talleres en cuanto a una línea de producción, como es el caso de las materas. Finalmente, en Ráquira, el 60\% (18 talleres) utiliza el $100 \%$ de su capacidad instalada, identificándose como pequeñas unidades de producción. Un 40\% (12 talleres) necesita recursos para poder aprovechar la capacidad productiva existente, ya sea por la falta de consecución de materias primas o el acondicionamiento del taller para una línea determinada de productos.

Descripción del subsector cerámico, alfarero en La Chamba - Tolima: En la medida en que el sector artesanal se transforma en una actividad empresarial, se requiere de economías de escala que permitan alcanzar los volúmenes necesarios y las calidades requeridas por los comercializadores. Esto ha implicado un lento proceso de especialización, lo cual se ve reflejado en la necesidad que han tenido de contratar en promedio dos empleados por taller (Soto, 1989). Así mismo, existe una división de trabajo femenino por edad, de tal manera que la fase inicial o el modelado es realizado por mujeres adultas, mientras que el proceso del brillado, lo efectúan en su mayoría, mujeres de poca edad. Esta táctica, se ha constituido en una forma de relacionar a las personas jóvenes con la producción y fomentar el aprendizaje.
El aumento en la demanda de productos artesanales de La Chamba ha generado mayores ingresos para algunos talleres, así que la actividad exportadora implica, en algunos casos, una mejora en la calidad de vida. Sin embargo, como se consignó en el Cuadro 3, que muestra los ingresos de los talleres encuestados, solamente uno de ellos muestra ingresos de ocho S.M.L. mensuales, mientras que el $44 \%$ de los talleres se conforman con un S.M.L. y, probablemente, dependen de otras actividades para sobrevivir.

Es importante recalcar que en La Chamba, hay algunos artesanos que cuentan con un mayor nivel de escolaridad que en Ráquira. Lo anterior implica que ellos comienzan a darle importancia a la educación como parte esencial de la formación de sus hijos, para tener "un mejor futuro y mayores oportunidades". Por lo anterior, quizás algunos de estos nuevos artesanos se convertirán en una generación distinta, con nuevas ideas, generando sinergias entre el artesano tradicional "sus padres" y los nuevos artesanos que cuentan con conocimientos técnicos para mejorar los procesos de producción, de comercialización y de administración.

La experiencia empírica unida al conocimiento de los artesanos jóvenes da excelentes resultados. Es el caso de la artesana Liliana Betancourt que estudió siete semestres de contaduría, y quién combina su formación con la de su madre que lleva 58 años en el oficio de artesana. Ellas se han convertido en líderes, generando empleo 
Cuadro 3. Ingresos de los artesanos medidos en Salario Mínimo Legal (S.M.L.) año 2004 (La Chamba).

\begin{tabular}{|c|c|c|c|}
\hline \multirow{2}{*}{ S.M.L.* } & \multirow{2}{*}{$\begin{array}{c}\text { Número de } \\
\text { Talleres }\end{array}$} & SI & NO \\
\cline { 3 - 4 } & 11 & 10 & 1 \\
\hline 1 S.M.L. & 4 & 4 & \\
\hline 1,5 S.M.L. & 5 & 5 & \\
\hline 2 S.M.L. & 4 & 4 & \\
\hline 2,5 S.M.L. & 1 & 1 & \\
\hline 8 S.M.L. & 25 & 25 & \\
\hline TOTALES & & &
\end{tabular}

Encuesta U.D.C.A, 2004

a través de un modelo de asociatividad promovido por la comunidad de artesanas que, conjuntamente con Artesanías de Colombia, les está ayudando a promover sus productos en el exterior.

En cuanto a los canales de distribución ocurre lo mismo que en Ráquira, es decir, en su mayoría los intermediarios comercializan los productos. Por este hecho, el $76 \%$ de las artesanas les venden sus productos en el mismo lugar de trabajo, haciendo énfasis en las facilidades existentes para este proceso. Un 15\% de artesanos (esposos), se dedican a la comercialización y para ello se trasladan a diferentes lugares del país a participar en ferias artesanales, representando para ellos mayor utilidad que venderlos en la misma comunidad. Se debe tener en cuenta que algunos de los intermediarios presionan a las artesanas a que les dejen el producto a crédito por 30 y hasta 60 días, lo cual disminuye las utilidades esperadas y el pago oportuno de los productos. Es importante mencionar la dificultad de las artesanas para calcular los precios de venta y su margen de utilidad, el mismo que no llega a imponerse en el momento de la operación comercial. Por lo anterior, la determinación de los precios está beneficiando a los intermediarios y a los dueños de almacenes, más no a los productores. Se destaca que el precio de venta al por menor en el punto de venta en almacenes especializados tiene un aumento de hasta el $80 \%$ en comparación con el precio de compra a los artesanos en el punto de producción. De allí, se deduce la necesidad de implementar un programa de comercialización, creando precios de sustentación, que beneficien a todos los artesanos y, además, incentivar el mejoramiento de la calidad de los productos.

Algunos artesanos a través de empresas de comercialización internacional están exportando sus productos bimensual o semestralmente. Para ello llega un contenedor hasta La Chamba a cargar los productos para evitar daños y deterioros en el envío. Igualmente, se está mejorando el empaque y elembalaje, los cuales con anterioridad eran hojas o afrechos del bosque, mientras que ahora se empacan de manera individual, lo cual protege y preserva la calidad, evitando pérdidas en el puerto de llegada. Sin embargo, se presentan averías cuando la aduana revisa la mercancía.

Las artesanías de la Chamba ya son conocidas en muchos países por su belleza y calidad. En el Cuadro 4, se observa que el $84 \%$ los talleres ya tiene pedidos para exportar. Se han creado modelos de asociatividad, pues un solo artesano no puede cumplir con la totalidad de los mismos. Los principales países destino de las exportaciones son Estados Unidos, Canadá, Grecia, Holanda, Alemania, Inglaterra, Israel y Panamá.

Cabe anotar que distintas cadenas de almacenes, como The Pottery, en la ciudad de Bogotá y Habitat, en Londres, les han hecho compras representativas, cuyas negociaciones han sido exitosas, teniendo en cuenta que en La Chamba sí ha sido posible cumplir con la demanda, ya que existe un buen nivel de asociatividad y compromiso por parte de los artesanos, quienes cumplen con los 
Cuadro 4. Potencial actividad exportadora de artesanías (La Chamba)

\begin{tabular}{|c|c|c|c|}
\hline \multirow{2}{*}{$\begin{array}{c}\text { PORCENTAJE DE } \\
\text { LA PRODUCCIÓN } \\
\text { DEDICADA A } \\
\text { EXPORTAR * }\end{array}$} & $\begin{array}{c}\text { Número de } \\
\text { Talleres }\end{array}$ & \multicolumn{2}{|c|}{ ACTIMDAD EXPORTADORA } \\
\cline { 3 - 4 } & & SI (INTERMEDIARIOS) & NO \\
\hline $0 \%$ & 4 & 4 & \\
\hline $10 \%$ & 1 & 1 & \\
\hline $50 \%$ & 3 & 3 & \\
\hline $80 \%$ & 5 & 5 & \\
\hline $100 \%$ & 12 & 12 & \\
\hline TOTAL & 25 & 25 & \\
\hline
\end{tabular}

Encuesta U.D.C.A, 2004

*Compras hechas por comercializadores para exportar. Año 2004

pedidos a tiempo y con las calidades requeridas, caso contrario a lo sucedido en el municipio Ráquira.

La asociatividad ha sido viable entre grupos pequeños de 5 a 20 artesanos que conforman unidades productivas, pero no representa la mayor parte de los artesanos. Lo anterior, ha generado un alto grado de insatisfacción en quienes no se vincularon a la asociación. Falta mucho por hacer, pues aunque los beneficios esperados en la actualidad aún no son distribuidos para la mayoría de la población artesanal, este es un buen comienzo.

\section{Comportamiento de las exportaciones del subsector} cerámico y/o alfarero: Colombia exportó en el año 2000 aproximadamente 18 millones de dólares en artesanías. Según las estadísticas sobresalen con buena participación y crecimiento los subsectores tradicionales, como la cerámica, las estatuillas en madera y otros subsectores que incursionan con elevado crecimiento, como la bisutería, los productos de origen animal o vegetal y las manufacturas de vidrio. Esta situación asegura un reconocimiento de estos productos colombianos en los mercados internacionales, ya que son percibidos como productos "hechos a mano", de excelente calidad (Proexport, 2003).

Del total de exportaciones colombianas de artesanías, las exportaciones del subsector cerámico tienen una participación del 50,6\%. En 1998, fueron US \$8`576 millones de dólares, en 1999 fueron US \$8`690 millones y en el año 2000 tuvieron un valor de US \$9 475 , señalando un crecimiento sostenido entre 1998 y 2000. El principal destino de las exportaciones de artesanías colombianas en el año 2002 fue Estados Unidos, con US \$5’300.000,oo aproximadamente, seguido por Venezuela con US \$2'900.000,oo y la Unión Europea US $\$ 1^{\prime} 900.000,00$. Sin embargo, es importante resaltar la cantidad y valor de artesanías que tienen como destino las zonas francas del país, especialmente Cartagena (Ministerio de Comercio Industria y Turismo, 2002).

Dentro de los sectores de promoción de Proexport para exportación, la cerámica ocupa el renglón más importante con un aumento significativo año a año, tal como se observa en el Cuadro 5.

Uno de los factores que los artesanos y específicamente el subsector cerámico debe tener en cuenta para poder seguir aumentando su participación dentro de las exportaciones de Colombia es el aseguramiento de la calidad con normas internacionales. Esto se considera una urgente necesidad para las micro, pequeñas y medianas empresas. 
Cuadro 5. Exportaciones colombianas del subsector cerámico por posición arancelaria.

\begin{tabular}{|c|c|c|c|c|c|c|}
\hline $\begin{array}{c}\text { POSICIÓN } \\
\text { ARANCELARIA }\end{array}$ & DESCRIPCIÓN & $\begin{array}{l}\text { FOB } \\
\text { (US\$) } \\
1999\end{array}$ & $\begin{array}{l}\text { FOB } \\
\text { (US\$) } \\
2000\end{array}$ & $\begin{array}{l}\text { FOB } \\
\text { (US\$) } \\
2001\end{array}$ & $\begin{array}{l}\text { PARTICIP. } \\
(\%) \\
2001\end{array}$ & $\begin{array}{l}\text { FOB } \\
\text { (US\$) } \\
2002\end{array}$ \\
\hline$\underline{6912000000}$ & $\begin{array}{l}\text { VAJILLA Y DEMÁS } \\
\text { ART ÍCULOS DE USO } \\
\text { DOMÉSTICO, HIGIENE O } \\
\text { TOCADOR EXCEPTO DE } \\
\text { PORCELANA }\end{array}$ & $5,429,848$ & $7,412,600$ & $7,157,607$ & 76.51 & $9,637,076$ \\
\hline$\underline{6913900000}$ & $\begin{array}{c}\text { LAS DEMÁS EST ATUILLAS Y } \\
\text { DEMÁS ART ÍCULOS PARA } \\
\text { ADORNO, DE CERÁMICA }\end{array}$ & $1,054,154$ & 960,353 & $1,120,506$ & 11.98 & $1,015,301$ \\
\hline$\underline{6914900000}$ & $\begin{array}{l}\text { LAS DEMÁS DE DEMÁS } \\
\text { MANUFACTURAS, DE } \\
\text { CERÁMICA }\end{array}$ & 758,118 & 477,194 & 508,097 & 5.43 & 517,090 \\
\hline$\underline{6911100000}$ & $\begin{array}{l}\text { ART ÍCULOS PARAEL } \\
\text { SERVICIO DE MESA O } \\
\text { COCINA, DE PORCELANA }\end{array}$ & $1,370,565$ & 362,940 & 449,358 & 4.80 & 780,163 \\
\hline$\underline{6913100000}$ & $\begin{array}{c}\text { EST ATUILLAS Y DEMÁS } \\
\text { OBJETOS DE ADORNO, DE } \\
\text { PORCELANA }\end{array}$ & 3,007 & 95,356 & 95,204 & 1.02 & 111,275 \\
\hline$\underline{6911900000}$ & $\begin{array}{l}\text { LOS DEMÁS, VAJILLAS Y } \\
\text { DEMÁS ART ÍCULOS DE } \\
\text { USO DOMÉSTICO, HIGIENE } \\
\text { O TOCADOR, DE } \\
\text { PORCELANA }\end{array}$ & 6,439 & 102,248 & 19,628 & 0.21 & 79,972 \\
\hline$\underline{7013100000}$ & $\begin{array}{l}\text { ART ÍCULOS DE } \\
\text { VITROCERÁMICA }\end{array}$ & 66,917 & 31,709 & 4,541 & 0.05 & 70 \\
\hline$\underline{6914100000}$ & $\begin{array}{l}\text { LAS DEMÁS } \\
\text { MANUFACT URAS, DE } \\
\text { PORCELANA }\end{array}$ & 169 & 39,516 & 40 & - & 73,579 \\
\hline \multicolumn{2}{|r|}{ TOTAL } & $8,689,217$ & $9,481,916$ & $9,354,981$ & 100 & $12,214,526$ \\
\hline
\end{tabular}

Fuente: Proexport 


\section{CONCLUSIONES}

La caracterización y el análisis del subsector reveló que no se practica la asesoría continua y progresiva para los artesanos, aunque sí han recibido ayudas técnicas en cuanto a capacitación, desde hace algunos años, por parte de entidades del Estado. En la zona rural, la actividad artesanal constituye la mayor fuente de ingresos y este oficio ha experimentado, en los últimos años, un aumento en el porcentaje de empleo generado, especialmente en la elaboración de cerámica. Existe una gran zona de influencia de producción artesanal que puede ser fácilmente aprovechada, destacándose por la diversificación de los productos.

La presencia de los comerciantes mayoristas, teóricamente favorece al artesano por el impulso que le da la comercialización en la zona, pero en la práctica, actúan solamente resguardando sus intereses. Esto implica que el artesano se siente desamparado ante las formas de trabajo aplicadas por los comerciantes mayoristas.

La comercialización de los productos artesanales, se facilita debido a la presencia de comerciantes-intermediarios y al desplazamiento de algunos artesanos a participar en ferias y la presencia de algunos almacenes ubicados en la comunidad. Sin embargo, el problema mayor es la ausencia de precios de sustentación, ya que los dueños de almacenes y comerciantes intermediarios los determinan para su bien, perjudicando a las artesanas que tienen un bajo nivel de producción. Finalmente, por influencia negativa de este gremio existe una tendencia a disminuir la calidad de los productos, básicamente por la orientación dada a algunos de ellos, en la que interviene activamente el comerciante mayorista, quien es portador de los requerimientos del mercado.

Se destaca la necesidad de recursos monetarios enfocados hacia diferentes aspectos, que faciliten la realización de las actividades artesanales. La poca capacitación de los artesanos en temas administrativos y económicos, así como su desconfianza en los programas de fomento, le impide atreverse a tomar créditos y aprovechar las alternativas propuestas por entidades financieras. Los artesanos no cuentan con los conocimientos suficientes para controlar sus actividades, por lo que son pocos los que llevan un control sobre la producción.
Existe una fuerte desconfianza y recelo por parte de los artesanos hacia las formas de trabajo asociativo o de organización y se observa una gran dificultad en lo que respecta a la mano de obra ocupada, principalmente por la falta de continuidad de los participantes en el proceso de producción, resaltando, por lo tanto, la participación de mano de obra en mayor porcentaje familiar que no familiar y con incidencia en la mayor cantidad de mujeres. Los ingresos que obtienen por esta actividad les ayuda a subsistir, no siendo considerada como la única fuente de ingresos, sino complementaria al aporte que hace el hombre para el sostenimiento de la familia.

Crece la analogía "Hecho a mano" es "Hecho con calidad", es decir, que los productos artesanales tienen un valor significativo en lo cultural, lo antropológico, lo sociológico y no solamente representa un valor económico para los consumidores de otros países. En un mundo globalizado, los consumidores de otros países aprecian nuestras artesanías, es decir, que hay un mercado potencial a nivel internacional que no está siendo aprovechado por los artesanos de los municipios de Ráquira y La Chamba.

\section{RECOMENDACIONES}

Realizar cursos de capacitación en aspectos relacionados con la producción, así como estudiar formas de apoyo al artesano, no consideradas por los comerciantes mayoristas, para mejorar la oportunidad de la comercialización de sus productos e implementar sistemas de crédito que le permitan tener mayor nivel de producción.

Desarrollar programas motivacionales para demostrar cuan importante es la actividad artesanal y crear estrategias de comercialización internacional de los productos artesanales, a través de nuevos sistemas de producción, teniendo en cuenta diseños originales y nuevas tecnologías que permitan ingresar y mantenerse dentro de los mercados internacionales.

Intensificar los programas de fomento que se desarrollan en la actualidad, con el objeto de inducir y motivar a la juventud en la práctica de las actividades artesanales y otorgar capacitación a los artesanos en temas administrativos y organizacionales que les ayude a establecer mecanismos de funcionamiento y a mantener un constante interés en su desarrollo y evaluación. 
Desarrollar un modelo que combine el proceso de producción y de comercialización a escala, para poder construir un modelo de comercialización internacional que permita aumentar la oferta exportable de productos cerámicos y/o alfareros, con un alto impacto en la generación de empleo y la consecución de divisas, con el fin de mejorar el nivel de vida de los artesanos del subsector en Colombia.

\section{BIBLIOGRAFÍA}

ARTESANÍAS DE COLOMBIA. 1999. Guía para exportar artesanías e identificación del oficio para la determinación de origen en Colombia. Bogotá: Ministerio de Desarrollo Económico, 185p.

BENAVIDES, E.O. 1997. Comportamiento de las exportaciones de las Artesanías Colombianas, 1972 1992. Santafé de Bogotá, Artesanías de Colombia S.A. 280 p.

DELGADO, T.C. 2001. Empaques para exportación de artesanías, del municipio de Guacamayas. Santafé de Bogotá: Universidad de los Andes, 250p.

ESTAGE, N.C. 1998. Artesanías mexicanas frente a la internacionalización. Revista Oaxaca 20:15.

FONSECA SARAVIA, J.M. 1985. Estudio de factibilidad de una empresa productora y comercializadora de artesanías para exportación. Santafé de Bogotá: Universidad de los Andes. 287p.

MINISTERIO DE DESARROLLO ECONÓMICO. 1998 a. Gestión institucional artesanías de Colombia 1990-
1998: una huella en el desarrollo artesanal. Santafe de Bogotá: Artesanías de Colombia, p.61.

MINISTERIO DE COMERCIO, INDUSTRIA Y TURISMO. 2002. Estadísticas. p.30.

NAVARRO G., P. 2001. Soportes y accesorios para artesanías de la Chamba a partir de material reciclado y biodegradable, Bogotá: Universidad de los Andes. p180.

PROEXPORT. 2003. Análisis del comportamiento de las exportaciones colombianas 1999 - 2002. p16.

RODRÍGUEZ G., M. 2001. Creación de nuevas empresa: artesanías colombianas para exportación a Canadá. Bogotá: Universidad de los Andes, 257p.

ROJAS E., G.1984. Diagnóstico de las necesidades del sector artesanal la Chamba, (Tolima) Santafé de Bogotá: Artesanías de Colombia, p.42.

SOTO P., C.C. 1989. Carpeta oficio artesanal, la Chamba, Tolima. Santafé de Bogotá á: Artesanías de Colombia, 1989. Dactilografiado, 158p.

URRUTIA, M. 1971. El sector artesanal en el desarrollo colombiano. Bogotá: Universidad Nacional. $178 p$.

VILLAREAL I., P. 2000. Centro de acopio de artesanías para exportación. Santa Fe de Bogotá: Universidad de los Andes, 350p.

Recibido: Julio 19 de 2006

Aceptado: Febrero 28 de 2007 